\title{
Real-time embedded system of super twisting-based integral sliding mode control for quadcopter UAV
}

\author{
Ahmad Riyad Firdaus \\ Electrical Engineering Department \\ Politeknik Negeri Batam \\ Batam, Indonesia \\ rifi@polibatam.ac.id
}

\author{
M. O. Tokhi \\ School of Engineering \\ London South Bank University \\ London, United Kingdom \\ tokhim@1sbu.ac.uk
}

\begin{abstract}
This paper presents the development of set-point weighting-based integral super-twisting sliding mode control (SISTASMC) with full-order state observers to overcome the control challenges encountered with nonlinear and underactuated systems. Quadcopter UAV form is a good example of underactuated systems, and this is selected in this research for validating the developed control. A comparative assessment through experimental validation is conducted between SISTASMC and Set-point weighting-based Integral Sliding Mode Control to demonstrate the performance of both controllers. Based on predetermined performance criteria, the results obtained demonstrate good performance of SISTASMC in dealing with uncertainty.

Index Terms-nonlinear and underactuated system, uncertainty, super-twisting algorithm, integral sliding mode control, setpoint weighting function.
\end{abstract}

\section{INTRODUCTION}

Various controllers have been developed for performance enhancement of quadcopter UAVs. Examples include proportional integral derivative (PID) control [1]; linear quadratic regulator (LQR) [2]; model predictive control (MPC) [3]; and sliding mode control (SMC) [4]. Among these, The SMC method outperforms others in terms of dealing with nonlinearity, underactuation, and uncertainty. This is because the controller is designed to be insensitive to such issues [5]. However, the chattering phenomenon following the use of SMC is still an issue to be resolved. Some methods have been proposed to eliminate this phenomenon. These include quasi sliding mode control (QSMC) [6]; interval type-2 fuzzy sliding mode control (IT2FSMC) [7]; high order sliding mode control (HOSMC) [8]; super-twisting algorithm of sliding mode control (STASMC) [9]; and dynamic sliding mode control (DSMC) [10]. Among the methods, STASMC has a better way to deal with uncertainty and disturbance.

This paper proposes performance enhancement of STASMC by employing integral term and set-point weighting function to the method to reduce steady-state error and overshoot. In addition, this paper presents experimental validation of the proposed controller and comparative performance assessment with QSMC.

This work was supported by Indonesia Endowment Fund for Education, Ministry of Finance Republic of Indonesia, and Politeknik Negeri Batam, Indonesia.
The rest of the paper is structured as follows: Section 2 describes dynamic model of the quadcopter, Section 3 presents control and observer design, experimental setup and results are presented in section 4 and 5 respectively. The paper is concluded in Section 6.

\section{QuAdCoPTER DyNAMic SYSTEM}

A quadcopter, as illustrated in Fig. 1, is a multirotor helicopter that is driven and lifted by four motors with total forces of $F_{i}(i=1,2,3,4)$.

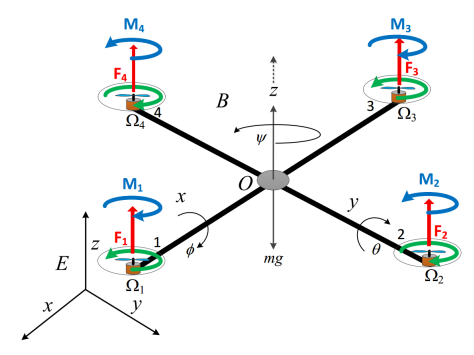

Fig. 1. Quadcopter UAVs frame work

In general, quadcopters utilize two pairs of twin fixed propellers; two clockwise $(1,3)$ and two counterclockwise $(2,4)$. These apply independent variation of the speed of each rotor to accomplish control. By changing the speed of each rotor it is possible to produce a desired total thrust; to locate for the centre of thrust both laterally and longitudinally; and to generate a desired total torque, or turning force.

The dynamical model of quadcopter can be expressed as

$$
\begin{gathered}
\ddot{x}=\frac{1}{m}(\cos \phi \cdot \sin \theta \cdot \cos \psi+\sin \phi \cdot \sin \psi) u_{1}-\frac{k_{1}}{m} \dot{x} \\
\ddot{y}=\frac{1}{m}(\cos \phi \cdot \sin \theta \cdot \sin \psi-\sin \phi \cdot \cos \psi) u_{1}-\frac{k_{2}}{m} \dot{y} \\
\ddot{z}=\frac{1}{m} \cos \phi \cdot \cos \theta \cdot u_{1}-g-\frac{k_{3}}{m} \dot{z} \\
\ddot{\phi}=\dot{\theta} \dot{\psi}\left(\frac{I_{y}-I_{z}}{I_{x}}\right)+\frac{l}{I_{x}} u_{2}-\frac{k_{4} l}{I_{x}} \dot{\phi} \\
\ddot{\theta}=\dot{\psi} \dot{\phi}\left(\frac{I_{z}-I_{x}}{I_{y}}\right)+\frac{l}{I_{y}} u_{3}-\frac{k_{5} l}{I_{y}} \dot{\theta} \\
\ddot{\psi}=\dot{\phi} \dot{\theta}\left(\frac{I_{x}-I_{y}}{I_{z}}\right)+\frac{C}{I_{z}} u_{4}-\frac{k_{6}}{I_{z}} \dot{\psi}
\end{gathered}
$$


where $\phi, \theta, \psi$ represent the angles of roll,pitch, and yaw, while $k_{i}$ represent the coefficients of drag and positive constants. $I_{x}, I_{y}, I_{z}$ denote the inertia of rotorcraft in $x, y, z$ axes respectively. The control inputs of quatcopter are represented by $u_{i}$ for total thrust of rotorcraft in $z$-axis, and $u_{2}, u_{3}$, and $u_{4}$ for roll, pitch, and yaw respectively. The relation between control input and forces generated by the propeller $\left(F_{i}\right)$ are expressed as

$u_{1}=\left(F_{1}+F_{2}+F_{3}+F_{4}\right)$

$u_{2}=\left(-F_{2}+F_{4}\right)$

$u_{3}=\left(-F_{1}+F_{3}\right)$

$u_{4}=d\left(-F_{1}+F_{2}-F_{3}+F_{4}\right) / b$

where $b$, and $d$ represent the lift coefficient and the scaling factor of force to moment.

\section{Control and Observer Design}

In this work, Set-point weighting-based Integral SuperTwisting Algorithm Sliding Mode Control (SISTASMC) is employed to control the altitude and attitude of the quadcopter system. However, Sliding Mode Control requires all state information from the plant for control requirement. Therefore, it is needed state observer to estimate umneasured states from plant. Furthermore, Quasi-Sliding Mode Observer (QSMO) is applied to acquire such required states. Meanwhile for control performance comparison, Quasi Set-point weightingbased Integral Sliding Mode Control (QSISMC) is used also to control attitude and altitude of quadcopter UAV. Generally, the overall control system is shown as in Fig. 2.

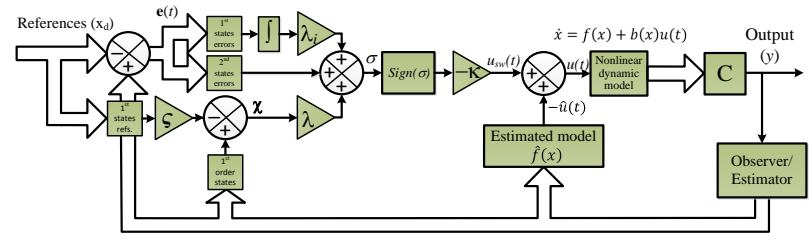

Fig. 2. Block diagram of overall control system

\section{A. Control Method}

Sliding mode control is a prominent robust control approach which gives an efficient way to deal with the problem of keeping up stability and consistent performance in the face of modelling imperfection. In order to account for the presence of disturbance and imperfection model, the control law should be discontinuous across $\sigma(t)$. However, this will result in chattering as seen in Fig. 3 due to the imperfection implementation of such control law. This phenomenon is not expected in practice because it involves high control activities.

One of the ways to address chattering issue is by developing Super-twisting algorithm of sliding mode control.

\section{B. Integral Slding Surface Design}

General form of nonlinear system can be defined as

$$
\begin{aligned}
& \dot{x}_{1}=x_{2} \\
& \dot{x}_{2}=f(\mathbf{x}, t)+b(\mathbf{x}, t) u(t)+d(t) \\
& y=x_{1}
\end{aligned}
$$

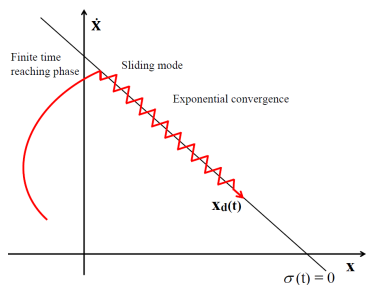

Fig. 3. Chattering phenomenon on the sliding surface

where $\mathbf{x}=\left[\begin{array}{ll}x_{1} & x_{2}\end{array}\right]$ is the state vector, $x_{1}$ and $x_{2}$ are system states, $y$ is output, $u(t)$ is control input, $f(\mathbf{x}, t)$ and $b(\mathbf{x}, t)$ represent general nonlinear functions. These functions are not accurately known, but the degree of inaccuracy on those function are upper bounded by a known continuous function of $\mathbf{x} \cdot d(t)$ is uncertain, and $|d(t)| \leqslant D_{0},|\dot{d}(t)| \leqslant D$. The control input role is to convey the state $\mathbf{x}$ to track a desired state $\mathbf{x}_{d}$ despite of model imperfection on $f(\mathbf{x}, t)$ and $b(\mathbf{x}, t)$.

A time varying integral switching surface $s(t)$ is defined in the state space $\mathbf{R}^{n}$ by equating the variable $s(\mathbf{x} ; t)$ to zero as expressed,

$$
s(\mathbf{x} ; t)=\left(\frac{d}{d t}+c\right)^{n-1} \mathbf{e}(t)+\lambda_{i} \int_{0}^{\infty} \mathbf{e}(t) d t
$$

where, $c, \lambda_{i}>0$ must be Hurwitz, taken to be the bandwidth of the system, and $\mathbf{e}(t)=\mathbf{y}(t)-\mathbf{x}_{d}(t)$ is the error in the output state, while $\mathbf{x}_{d}(t)$ is the expected state. An integral term of tracking error is introduced into equation to reduce steadystate errors.

\section{Set-point Weighting Function}

Employing integral term in SMC is believed to improve the controller performance in reducing steady-state error $\left(e_{s s}\right)$. However, any of the following issues can generate an overshoot in output variable:

- $|\mathbf{e}(t)|>\left|\mathbf{e}_{s s}\right|$, where $\mathbf{e}_{s s}$ is steady-state error of $\mathbf{e}(t)$,

- $\frac{\mathbf{e}(t)}{|\mathbf{e}(t)|} \neq \frac{\mathbf{e}_{s s}}{\left|\mathbf{e}_{s s}\right|}$

Additionally, considering typical feedback controller design, such as PID, the issue will be how to achieve fast reference tracking and reject disturbance at the same time. Generally, high-gain control parameters are required to deal with load disturbance. However at the same time, it will lead to oscillation or high overshoot of step response. This problem can be solved by introducing set-point weighting to increase the frequency of Zero, and subsequently diminish the overshoot in the output [11].

This approach is adopted in this work to handle the problem with integral SMC to reduce overshoot in output states while maintaining robustness of the controller. Therefore, the new sliding mode approach by applying set-point weighting can be designed as shown in Fig. 2. Accordingly, a new error $\chi(t)$ for proportional action is presented as

$$
\chi=e(t)+r(1-\varsigma)
$$

where $r$ is reference and $\varsigma$ is constant between 0 and 1 . Reducing $\varsigma$ will lead to decreasing overshoot and rise time, and 
vice versa. Consequently, the new sliding surface is defined as,

$$
s(\mathbf{x} ; t)=\left(\frac{d}{d t}+c\right)^{n-1} \chi(t)+\lambda_{i} \int_{0}^{\infty} \mathbf{e}(t) d t
$$

\section{Control law}

Furthermore, the SISTASMC for perturbation and chattering attenuation is defined by

$$
\begin{aligned}
& u_{s w}=-k_{1} \sqrt{|s|} \operatorname{sign}(s)+v \\
& \dot{v}=-k_{2} \operatorname{sign}(s)
\end{aligned}
$$

A suitable method for tuning its parameters is the accompanying pair of relationships

$$
\begin{aligned}
& k_{1}=\sqrt{\kappa} \\
& k_{2}=1.1 \kappa
\end{aligned}
$$

where $\kappa>0$ is chosen to be adequately large.

Therefore, the total control signal for nonlinear systems is expressed as,

$$
u(x, t)=\frac{1}{\hat{b}(x, t)}\left(\hat{u}(x, t)+u_{s w}\right)
$$

where $\hat{u}(x, t)$ for each degree of freedom is defined as,

$$
\begin{aligned}
& \hat{u}_{x}=-\mu_{x} s_{x}+\ddot{x_{d}}-\lambda_{x} \dot{e_{x}}-\lambda_{i x} e_{x} \\
& \hat{u}_{y}=-\mu_{y} s_{y}+\ddot{y_{d}}-\lambda_{y} \dot{e_{y}}-\lambda_{i y} e_{y} \\
& \hat{u}_{z}=-\mu_{z} s_{z}-\lambda_{z} \dot{e}_{z}-\lambda_{i z} e_{z}+\ddot{z}_{d}+g \\
& \hat{u}_{\phi}=-\mu_{\phi} s_{\phi}-\lambda_{\phi} \dot{e}_{\phi}-\lambda_{i \phi} e_{\phi}-\frac{\dot{\theta} \dot{\psi}}{I_{x}}\left(I_{y}-I_{z}\right)+\ddot{\phi}_{d} \\
& \hat{u}_{\theta}=-\mu_{\theta} s_{\theta}-\lambda_{\theta} \dot{e}_{\theta}-\lambda_{i \theta} e_{\theta}-\frac{\dot{\phi} \dot{\psi}}{I_{y}}\left(I_{z}-I_{x}\right)+\ddot{\theta_{d}} \\
& \hat{u}_{\psi}=-\mu_{\psi} s_{\psi}-\lambda_{\psi} \dot{e}_{\psi}-\lambda_{i \psi} e_{\psi}-\frac{\dot{\phi} \dot{\theta}}{I_{z}}\left(I_{x}-I_{y}\right)+\ddot{\psi_{d}}
\end{aligned}
$$

Meanwhile, the switching controls are defined as,

$$
\begin{aligned}
& u_{s w x}=-k_{1 x} \sqrt{\left|s_{x}\right|} \operatorname{sign}\left(s_{x}\right)-k_{2 x} \int_{0}^{\infty} \operatorname{sign}\left(s_{x}\right) d t \\
& u_{s w y}=-k_{1 y} \sqrt{\left|s_{y}\right|} \operatorname{sign}\left(s_{y}\right)-k_{2 y} \int_{0}^{\infty} \operatorname{sign}\left(s_{y}\right) d t \\
& u_{s w z}=-k_{1 z} \sqrt{\left|s_{z}\right|} \operatorname{sign}\left(s_{z}\right)-k_{2 z} \int_{0}^{\infty} \operatorname{sign}\left(s_{z}\right) d t \\
& u_{s w \phi}=-k_{1 \phi} \sqrt{\left|s_{\phi}\right|} \operatorname{sign}\left(s_{\phi}\right)-k_{2 \phi} \int_{0}^{\infty} \operatorname{sign}\left(s_{\phi}\right) d t \\
& u_{s w \theta}=-k_{1 \theta} \sqrt{\left|s_{\theta}\right|} \operatorname{sign}\left(s_{\theta}\right)-k_{2 \theta} \int_{0}^{\infty} \operatorname{sign}\left(s_{\theta}\right) d t \\
& u_{s w \psi}=-k_{1 \psi} \sqrt{\left|s_{\psi}\right|} \operatorname{sign}\left(s_{\psi}\right)-k_{2 \psi} \int_{0}^{\infty} \operatorname{sign}\left(s_{\psi}\right) d t
\end{aligned}
$$

Therefore, from (14) and (15), the control input for quadcopter UAVs can be presented as,

$$
\begin{aligned}
& u_{1}=\frac{m}{c \phi c \theta}\left(u_{s w z}+\hat{u}_{z}\right) \\
& u_{2}=\frac{I_{x}}{l}\left(u_{s w \phi}+\hat{u}_{\phi}\right) \\
& u_{3}=\frac{I_{y}}{l}\left(u_{s w \theta}+\hat{u}_{\theta}\right) \\
& u_{4}=I_{z}\left(u_{s w \psi}+\hat{u}_{\psi}\right)
\end{aligned}
$$

The calculation of the desired pitch $\left(\theta_{d}\right)$ and roll $\left(\phi_{d}\right)$ angles with $x$ and $y$ axes errors is given as,

$$
\begin{gathered}
\theta_{d}=\operatorname{atan}\left(\frac{\left(u_{s w x}+\hat{u}_{x}\right) c \phi c \theta}{u_{s w z}+\hat{u}_{z}}\right) \\
\phi_{d}=-\operatorname{atan}\left(\frac{\left(u_{s w y}+\hat{u}_{y}\right) c \phi c \theta}{u_{s w z}+\hat{u}_{z}}\right)
\end{gathered}
$$

\section{E. Observer Design}

QSMO, as a nonlinear estimator, has interesting properties in that the capacity to create a sliding motion on the estimation error, and to generates estimated states precisely equivalent with actual output of the plant. In addition, this method can eliminate chattering issue in SMO approach.

Before designing states observer for quadcopter, it is important to determine states vector of the system. Hence, the state vector of the system is defined as,

$$
\begin{aligned}
& x_{1}=\phi ; x_{2}=\dot{\phi} ; x_{3}=\theta ; x_{4}=\dot{\theta} ; x_{5}=\psi ; x_{6}=\dot{\psi} \\
& x_{7}=z ; x_{8}=\dot{z} ; x_{9}=x ; x_{10}=\dot{x} x_{11}=y ; x_{12}=\dot{y}
\end{aligned}
$$

In this case, the measured states of the rotorcraft are $\operatorname{roll}(\phi)$, pitch $(\theta)$ and $y a w(\psi)$ angles, and movements in $x, y, z$ axes. Moreover, the observer plays a critical role in estimating the velocity of the rotorcraft, such as $x_{2}, x_{4}, x_{6}, x_{8}, x_{10}$, and $x_{12}$. Thus, the QSMO for the quadcopter model is presented as

$$
\begin{aligned}
& \dot{\hat{x}}_{1}=-\alpha_{1} e_{1}+\hat{x}_{2}-k_{1} \frac{e_{1}}{\left|e_{1}\right|+\varepsilon} \\
& \dot{\hat{x}}_{2}=-\alpha_{2} e_{1}+\hat{x}_{4} \hat{x}_{6}\left(\frac{I_{y}-I_{z}}{I_{x}}\right)+\frac{l}{I_{x}} u_{2}-k_{2} \frac{e_{1}}{\left|e_{1}\right|+\varepsilon} \\
& \dot{\hat{x}}_{3}=-\alpha_{1} e_{3}+\hat{x}_{4}-k_{1} \frac{e_{3}}{\left|e_{3}\right|+\varepsilon} \\
& \dot{\hat{x}}_{4}=-\alpha_{2} e_{3}+\hat{x}_{2} \hat{x}_{6}\left(\frac{I_{z}-I_{x}}{I_{y}}\right)+\frac{l}{I_{y}} u_{3}-k_{2} \frac{e_{3}}{\left|e_{3}\right|+\varepsilon} \\
& \dot{\hat{x}}_{5}=-\alpha_{1} e_{5}+\hat{x}_{6}-k_{1} \frac{e_{5}}{\left|e_{5}\right|+\varepsilon} \\
& \dot{\hat{x}}_{6}=-\alpha_{2} e_{5}+\hat{x}_{2} \hat{x}_{4}\left(\frac{I_{x}-I_{y}}{I_{z}}\right)+\frac{1}{I_{z}} u_{4}-k_{2} \frac{e_{5}}{\left|e_{5}\right|+\varepsilon} \\
& \dot{\hat{x}}_{7}=-\alpha_{1} e_{7}+\hat{x}_{8}-k_{1} \frac{e_{7}}{\left|e_{7}\right|+\varepsilon} \\
& \dot{\hat{x}}_{8}=-\alpha_{2} e_{7}-g+\frac{c x_{1} c x_{3}}{m} u_{1}-k_{2} \frac{e_{7}}{\left|e_{7}\right|+\varepsilon} \\
& \dot{\hat{x}}_{9}=-\alpha_{1} e_{9}+\hat{x}_{10}-k_{1} \frac{e_{9}}{\left|e_{9}\right|+\varepsilon} \\
& \dot{\hat{x}}_{10}=-\alpha_{2} e_{9}+\frac{c x_{1} s x_{3} c x_{5}+s x_{1} s x_{5}}{m} u_{1}-k_{2} \frac{e_{9}}{\left|e_{9}\right|+\varepsilon} \\
& \dot{\hat{x}}_{11}=-\alpha_{1} e_{11}+\hat{x}_{12}-k_{1} \frac{e_{11}}{\left|e_{11}\right|+\varepsilon} \\
& \dot{\hat{x}}_{12}=-\alpha_{2} e_{11}+\frac{c x_{1} s x_{3} s x_{5}-s x_{1} c x_{5}}{m} u_{1}-k_{2} \frac{e_{11}}{\left|e_{11}\right|+\varepsilon}
\end{aligned}
$$


where $\tilde{x}=\hat{x}-x$ is the estimation error of states, $\hat{x}, \alpha_{i}$ and $k_{i}$ represent estimated states, constants, and positive constants respectively.

\section{EXPERIMENTAL SETUP}

In this research, myRIO-1900 platform with LABVIEW based-framework, is used to apply the proposed controller and observer in real-time application. The mass moment of inertia, mass, and length of quadcopter arms are real parameters obtained by measurements. The parameters of the plant are as presented in Table I, meanwhile the selected parameter values for controllers are shown in Tables III and II. The saturation constant $(\epsilon)$ for QSISMC is 0.1 . To estimate unmeasured states, the selected observer parameters are presented as $\alpha_{1}=120, \alpha_{1}$ $=300, K_{1}=0.1$, and $K_{2}=5$. The selection of the controllers and observer parameters is carried out through trial and error (manual tune) until obtaining optimal performance from the two controllers and observer. Furthermore, the set-point weighting values $(\zeta)$ for roll $(\phi)$ are 1 and 0.83 , and for pitch $(\theta)$ are 1 and 0.82 . The selection of the two values of set-point weighting function $(\zeta)$ for each state is to demonstrate the effect of the weighting on the performance of the controller. In this experimental work, the sampling time $(t)$ for control and estimation process is 0.001 seconds.

TABLE I

QUADCOPTER PARAMETERS

\begin{tabular}{|c|c|c|}
\hline \multirow{2}{*}{ Variabels } & \multicolumn{2}{|c|}{ Values } \\
\cline { 2 - 3 } & Observer \& control models & Units \\
\hline$m$ & 1.79 & $\mathrm{~kg}$ \\
\hline$l$ & 0.29 & $\mathrm{~m}$ \\
\hline$I_{x}$ & 0.026281674 & $\mathrm{kgm}^{2}$ \\
\hline$I_{y}$ & 0.027484487 & $\mathrm{kgm}^{2}$ \\
\hline$I_{z}$ & 0.045603074 & $\mathrm{kgm}^{2}$ \\
\hline$g$ & 9.81 & $\mathrm{~m} / \mathrm{s}^{2}$ \\
\hline
\end{tabular}

TABLE II

CONTROLLER PARAMETERS FOR SISTASMC METHOD

\begin{tabular}{|c|c|c|c|c|c|c|c|}
\hline \multicolumn{1}{|c|}{ SISTASMC parameters } \\
\hline Var & Values & Var & Values & Var & Values & Var & Values \\
\hline$\lambda_{\phi}$ & 1.485 & $\lambda_{\theta}$ & 1.251 & $\lambda_{\psi}$ & 1.109 & $\lambda_{z}$ & 1 \\
\hline$\lambda_{i \phi}$ & 5.76 & $\lambda_{i \theta}$ & 5.76 & $\lambda_{i \psi}$ & 0.5 & $\lambda_{i z}$ & 0.1 \\
\hline$\kappa_{\phi}$ & 80.9 & $\kappa_{\theta}$ & 97.86 & $\kappa_{\psi}$ & 60 & $\kappa_{z}$ & 50 \\
\hline$\mu_{\phi}$ & 17.5 & $\mu_{\theta}$ & 17.5 & $\mu_{\psi}$ & 17.5 & $\mu_{z}$ & 17.5 \\
\hline
\end{tabular}

TABLE III

CONTROLLER PARAMETERS FOR QSISMC METHOD

\begin{tabular}{|c|c|c|c|c|c|c|c|}
\hline \multicolumn{1}{|c|}{ QSISMC parameters } \\
\hline Var & Values & Var & Values & Var & Values & Var & Values \\
\hline$\lambda_{\phi}$ & 1.485 & $\lambda_{\theta}$ & 1.251 & $\lambda_{\psi}$ & 1.109 & $\lambda_{z}$ & 1 \\
\hline$\lambda_{i \phi}$ & 5.76 & $\lambda_{i \theta}$ & 5.76 & $\lambda_{i \psi}$ & 0.5 & $\lambda_{i z}$ & 0.1 \\
\hline$k_{\phi}$ & 80.9 & $k_{\theta}$ & 97.86 & $k_{\psi}$ & 60 & $k_{z}$ & 50 \\
\hline$\mu_{\phi}$ & 17.5 & $\mu_{\theta}$ & 17.5 & $\mu_{\psi}$ & 17.5 & $\mu_{z}$ & 17.5 \\
\hline
\end{tabular}

Comparative assessment of the two controllers is carried out subject to various set-point weighting values. Maximum overshoot, rise time (Tr), and steady-state error (Ess) are taken into account to evaluate the performance of controllers and observer.
In this experiment, however, the number of states to be controlled is reduced to 8 due to the availability of adequate sensors, by excluding: $x$-movement, rate of $x$-movement $(\dot{x})$, $y$-movement, and rate of $y$-movement $(\dot{y})$. In addition, due to time constraints, the amount of data taken is only for 4 states, namely $\operatorname{roll}(\phi)$, rate of $\operatorname{roll}(\dot{\phi}), \operatorname{pitch}(\theta)$ and rate of $\operatorname{pitch}(\dot{\theta})$. The consideration of selecting such data is because the data is very crucial in the application of quadcopter UAV. Based on these considerations, the complete experimental validations for 12 states will be performed in future works.

\section{EXPERIMENTAL RESULTS}

\section{A. Roll movements}

Performance assessment through comparative studies between two controllers is investigated and analysed subject to set-point weighting values. Furthermore, to produce a more valid comparison analysis, data retrieval is done at a voltage level of 11.6 volts. The experimental results obtained are shown in Fig. 4 - 9

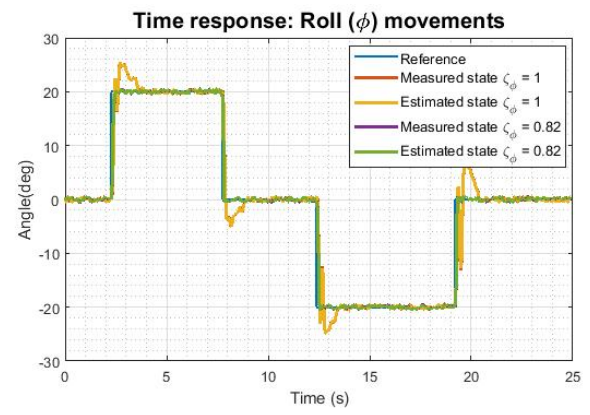

Fig. 4. Roll movements - SISTASMC

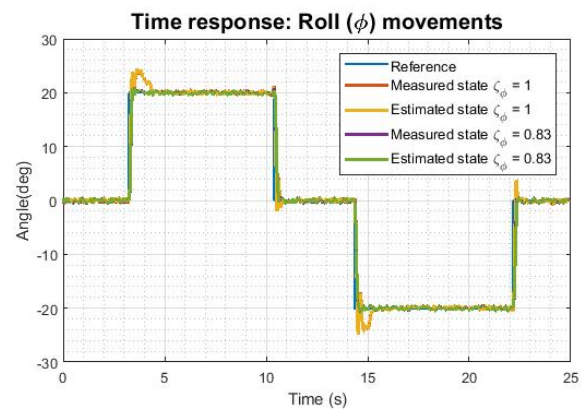

Fig. 5. Roll movements - QSISMC

As noted, although the reference changes suddenly, in general, the two controllers, namely SISTASMC and QSISMC showed good response to keep the system trajectory to track the reference with fast rise time and small tracking errors as seen in Fig. 4 - 5. Moreover, the chattering phenomenon following the issue of sliding mode control was reduced effectively by employing the two control methods so the overall performance of the system was not affected. The performance of the two controllers also improved after introducing set-point weighting values to the control system. It can be seen in Fig. 
4 - 5 and Table IV that the overshoot phenomenon due to the utilization of integral action in the control systems was reduced significantly by reducing the weighting value from $27.42 \%$ to $2.5 \%$ for SISTASMC and from $22.65 \%$ to $4.115 \%$ for QSISMC without affecting Tr significantly, and the Ess remained at similar values for both control systems. Fast rise time is demonstrated by the two controllers as shown in the Table IV, around 0.144 seconds $(\zeta=1)$ and 0.132 seconds $(\zeta=0.83)$ for SISTASMC; and 0.108 seconds $(\zeta=1)$ and 0.152 seconds $(\zeta=0.83)$ for QSISMC.

TABLE IV

CONTROL SYSTEMS PERFORMANCES

\begin{tabular}{|l|c|r|c|c|c|c|}
\hline \multirow{2}{*}{ Controls } & \multicolumn{2}{|c|}{ Overshoot (\%) } & \multicolumn{2}{c|}{$\operatorname{Tr}(\mathbf{s})$} & \multicolumn{2}{c|}{ Ess(deg) } \\
\cline { 2 - 7 } & $\zeta=\mathbf{1}$ & $\zeta=\mathbf{0 . 8 3}$ & $\zeta=\mathbf{1}$ & $\zeta=\mathbf{0 . 8 3}$ & $\zeta=\mathbf{1}$ & $\zeta=\mathbf{0 . 8 3}$ \\
\hline QSISMC & 22.65 & 4.115 & 0.108 & 0.152 & 0.226 & 0.229 \\
\hline SISTASMC & 27.42 & 2.5 & 0.144 & 0.132 & 0.247 & 0.205 \\
\hline
\end{tabular}

Noting the control signals in Fig. 6 - 7, in the practical application, high frequency oscillations were seen in the signals for both control systems. However, the oscillations were small. This phenomenon occurs due to maintaining the system trajectory on the reference for all times.

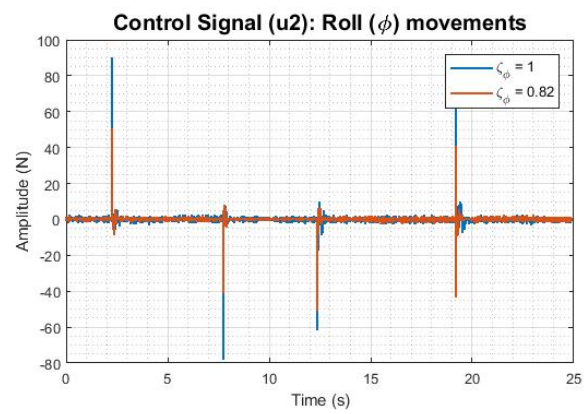

Fig. 6. Control signals - SISTASMC

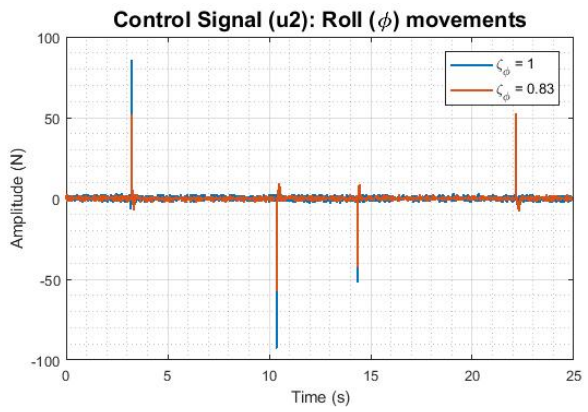

Fig. 7. Control signals - QSISMC

In most cases, all control systems succeeded in dealing with nonlinearity of the system and all unforeseen circumstances. However, observing Table IV, the two controllers showed slightly different performances. Although SISTASMC exhibited higher overshoot when the set-point weighting value was 1, SISTASMC showed smaller overshoot and steady-state errors compared with QSISMC when the weighting value was 0.83 .

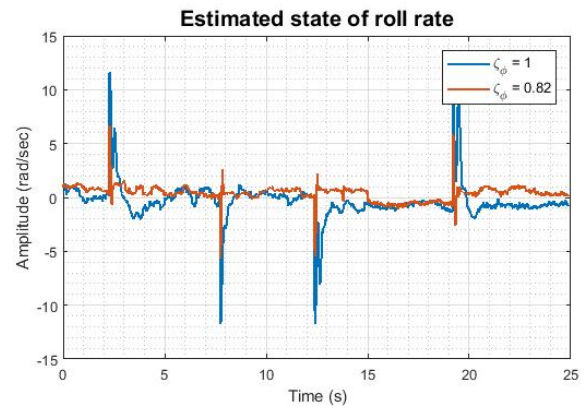

Fig. 8. Roll rate - SISTASMC

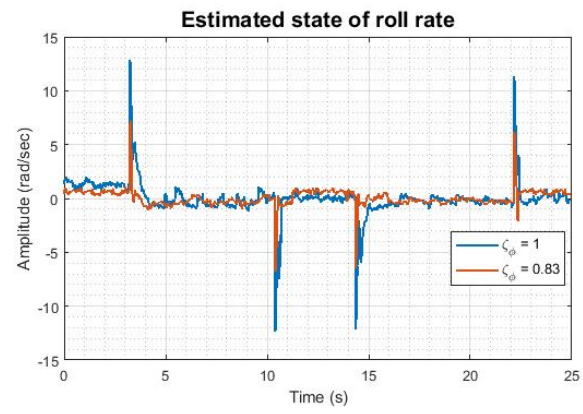

Fig. 9. Roll rate - QSISMC

In this experimental validations, the observer performed quite well as seen in Fig. 8 - 9. The observer provided proper unmeasured states to be utilized in the control systems. It can be seen in Table V that mean squared error (MSE) of estimated roll rate (ERR) in steady-state condition was quite small. Furthermore, the performance quality of the observer was evaluated by measuring estimation error (EE) of tracking true state. As shown in the table, the estimation errors were very small for all the controller methods. It means that the values of estimated states were very close to the actual ones.

TABLE V

OBSERVER PERFORMANCES

\begin{tabular}{|l|c|c|c|c|}
\hline \multirow{2}{*}{ Controls } & \multicolumn{2}{|c|}{ MSE EE } & \multicolumn{2}{c|}{ MSE ERR } \\
\cline { 2 - 5 } & $\zeta=\mathbf{1}$ & $\zeta=\mathbf{0 . 8 3}$ & $\zeta=\mathbf{1}$ & $\zeta=\mathbf{0 . 8 3}$ \\
\hline SISTASMC & $3.51 \mathrm{E}-06$ & $8.47465 \mathrm{E}-07$ & 0.399361504 & 0.54728051 \\
\hline QSISMC & $2.81804 \mathrm{E}-06$ & $9.61817 \mathrm{E}-07$ & 0.300457406 & 0.180575645 \\
\hline
\end{tabular}

Set-point weighting function also contributed to improving the performance of the observer as seen in the table. Overall, by introducing smaller weighting values to control the system, the performance of the observer improved significantly by showing smaller MSE of estimation error and MSE of estimated roll rate.

\section{B. Pitch movements}

The performance of controllers, including SISTASMC and QSISMC; and observer, namely QuasiSMO were also evaluated with free of payload disturbance to control pitch movements of the quadcopter UAV. Performance assessment through comparative studies between two controllers was 
investigated and analysed also subject to set-point weighting values. The same as previous works, to produce a more valid comparison analysis, data retrieval was done at a voltage level of 11.6 volts.

The experimental results obtained for pitch movements are presented in Fig. 10 - 15.

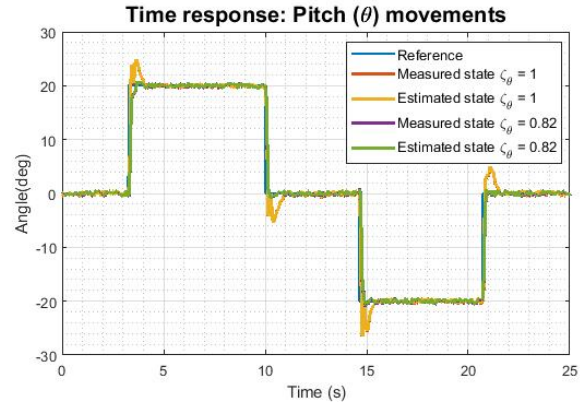

Fig. 10. Pitch movements - SISTASMC

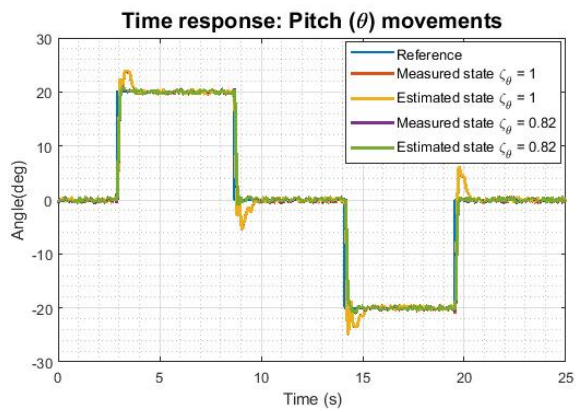

Fig. 11. Pitch movements - QSISMC

Similar with roll motions, in general, both controllers, namely SISTASMC and QSISMC showed good response to keep the system trajectory to track the reference with fast rise time and small tracking errors as seen in Fig. 10 11 despite of sudden changes of reference. Moreover, the chattering phenomenon following the issue of sliding mode control was reduced effectively by employing the two control methods so the overall performance of the system was not affected. The performance of the controllers was also improved after introducing set-point weighting values to the control systems. It can be seen in Fig. $10-11$ and Table VI that the overshoot phenomenon due to the utilization of integral action in the control systems was reduced significantly by reducing the weighting value from $24.53 \%$ to $4.155 \%$ for SISTASMC, and from $20.44 \%$ to $4.915 \%$ for QSISMC without affecting Tr significantly, and the Ess remained at similar value for both control systems. Fast rise time was achieved by the two controllers as shown in Table VI, around 0.128 seconds $(\zeta=1)$ and 0.200 seconds $(\zeta=0.82)$ for SISTASMC; and 0.148 seconds $(\zeta=1)$ and 0.164 seconds $(\zeta=0.82)$ for QSISMC

Observing the control signals in Fig. 12 - 13, in the practical application, high frequency oscillations were seen
TABLE VI

CONTROL SYSTEMS PERFORMANCES

\begin{tabular}{|l|c|c|c|r|c|r|}
\hline \multirow{2}{*}{ Controls } & \multicolumn{2}{|c|}{ Overshoot (\%) } & \multicolumn{2}{|c|}{$\mathbf{T r}(\mathbf{s})$} & \multicolumn{2}{c|}{ Ess(deg) } \\
\cline { 2 - 7 } & $\zeta=\mathbf{1}$ & $\zeta=\mathbf{0 . 8 2}$ & $\zeta=\mathbf{1}$ & $\zeta=\mathbf{0 . 8 2}$ & $\zeta=\mathbf{1}$ & $\zeta=\mathbf{0 . 8 2}$ \\
\hline QSISMC & 20.44 & 4.915 & 0.148 & 0.164 & 0.224 & 0.214 \\
\hline SISTASMC & 24.53 & 4.155 & 0.128 & 0.2 & 0.176 & 0.213 \\
\hline
\end{tabular}

in the signals for both control systems. However, this was small. This phenomenon occurs due to maintaining the system trajectory on the reference for all times.

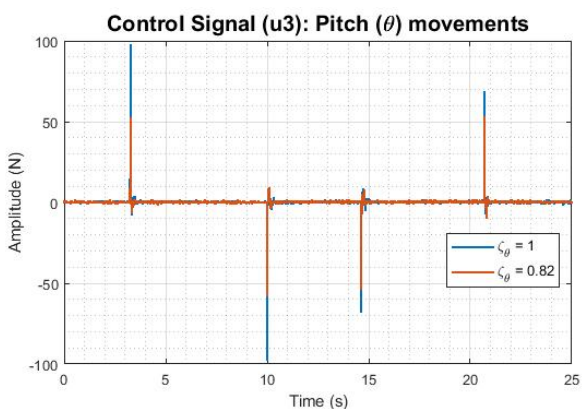

Fig. 12. Control signals - SISTASMC

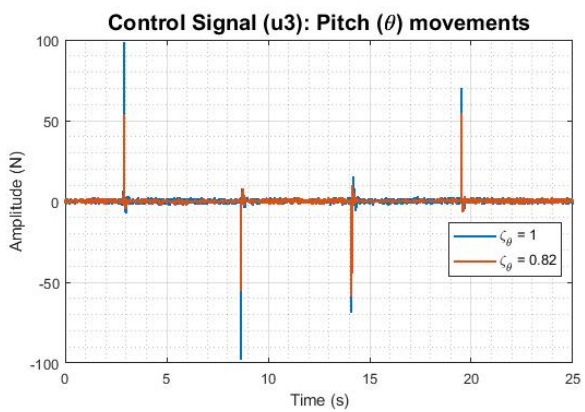

Fig. 13. Control signals - QSISMC

Generally, both control systems were successful in handling the nonlinearity of the system and all unforeseen circumstances. With reference to Table IV, the two controllers showed similar performances in controlling the pitch movements of the vehicle. There were slightly different performance results between SISTASMC and QSISMC for instant, SISTASMC had lower overshoot than QSISMC when $\zeta$ was 0.82 , and the controller had lower Ess for both $\zeta$ values, but the differences were not significant. Therefore, in this case, both controllers showed similar performances in controlling the pitch movements.

In this experimental validations, the observer has performed quite well as seen in Fig. 14 - 15 to estimate unmeasured states and tracking true states. The observer generated adequate pitch rate state to be utilized in the control systems. It can be seen in Table VII that MSE of estimated pitch rate (EPR) in steadystate condition was quite small. Furthermore, the performance quality of the observer was evaluated by measuring estimation error (EE) of tracking true states. As shown in the same 


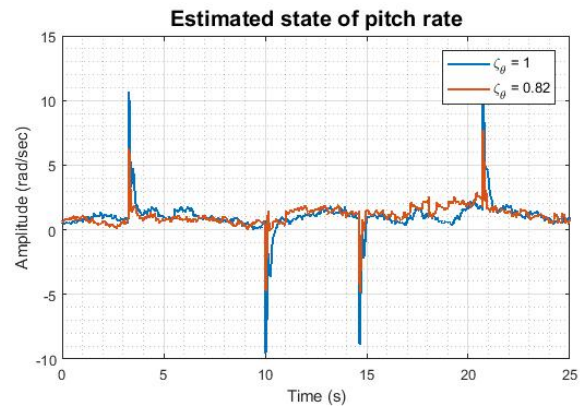

Fig. 14. Pitch rate - SISTASMC

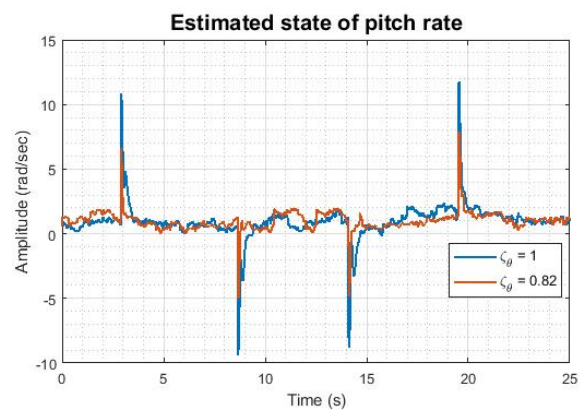

Fig. 15. Pitch rate - QSISMC

table the estimation errors were very small for all the control systems. It means that the values of estimated states were very close to the actual ones.

TABLE VII

OBSERVER PERFORMANCES

\begin{tabular}{|l|c|c|c|c|}
\hline \multirow{2}{*}{ Controls } & \multicolumn{2}{|c|}{ MSE EE } & \multicolumn{2}{c|}{ MSE EPR } \\
\cline { 2 - 5 } & $\zeta=\mathbf{1}$ & $\zeta=\mathbf{0 . 8 2}$ & $\zeta=\mathbf{1}$ & $\zeta=\mathbf{0 . 8 2}$ \\
\hline QSISMC & $2.6566 \mathrm{E}-06$ & $1.56151 \mathrm{E}-06$ & 0.485602409 & 0.560899811 \\
\hline SISTASMC & $2.38261 \mathrm{E}-06$ & $2.08375 \mathrm{E}-06$ & 1.089531314 & 0.586225678 \\
\hline
\end{tabular}

Furthermore, set-point weighting function also contributed to improving the performance of the observer as seen in Table VII. Overall, by introducing smaller weighting values into the control system, the performance of the observer improved significantly by showing smaller MSE of estimation error and MSE of estimated pitch rate.

\section{CONCLUSION}

From the experimental works, it can be summarized that both selected controllers, namely SISTASMC and QSISMC have shown good performance in dealing with the nonlinear and underactuated system as well as unforeseen circumstances, including: possibility of parameters mismatch. The issue of unavailability of information on system state has been handled very well by QuasiSMO as observer in this real-time application. The unforeseen circumstances have not affected much the performance of the observer. Furthermore, the presence of setpoint weighting function has played a vital role in improving the performance of the controllers by demonstrating good character in reducing overshoot significantly while maintaining rise time in small values. In addition, generally, by employing this weighting function, the Ess and estimation errors were reduced quite well.

In terms of controllers performance comparisons, in general, SISTASMC has shown better ability than QSISMC to control some movements of quadcopter UAV especially. In addition, the controller has been able to improve the observer's performance well so that some unmeasured states can be provided adequately and the estimation values of true state is very close to actual values. This fact proves that SISITASMC has a better ability to handle parameter uncertainties than QSISMC.

\section{ACKNOWLEDGMENT}

This work was supported by Indonesia Endowment Fund for Education, Ministry of Finance Republic of Indonesia, and Politeknik Negeri Batam, Indonesia.

\section{REFERENCES}

[1] Minhuan Guo, Yan Su, and Dongbing Gu, "System identification of the quadrotor with inner loop stabilisation system," International Journal of Modelling, Identification and Control, vol. 28, no. 3, 2017.

[2] Wesam Jasim and Dongbing Gu, "Iterative linear quadratic regulator control for quadrotors leader-follower formation flight," International Journal of Modelling, Identification and Control, vol. 31, no. 2, 2019.

[3] A. Zanelli and A. Domahidi and J. Jerez and M. Morari, "FORCES NLP: an efficient implementation of interior-point methods for multistage nonlinear nonconvex programs," International Journal of Control, vol. 0, no. 0, pp. 1-17, 2017.

[4] En-Hui Zheng, Jing-Jing Xiong, and Ji-Liang Luo, "Second order sliding mode control for a quadrotor UAV," ISA transactions, vol. 53, pp. 13506, 2014.

[5] Ahmad Riyad Firdaus and Arief Syaichu Rahman, "Genetic algorithm of sliding mode control design for manipulator robot," TELKOMNIKA (Telecommunication Computing Electronics and Control), vol. 10, no. 4, pp. 645-654, 2012.

[6] Yuri Shtessel, Christopher Edwards, Leonid Fridman, and Arie Levant, Sliding Mode Control and Observation, Springer-Verlag, New York, 2010.

[7] Ahmad Riyad Firdaus and M. O. Tokhi, "Sliding mode control-based interval type-2 fuzzy logic controller for quadcopter uavs," in ASSISTIVE ROBOTICS: Proceedings of the 18th International Conference on CLAWAR 2015, HangZhou China, September 2015, pp. 555-563.

[8] Jalel Ghabi, "A novel sliding mode controller scheme for a class of nonlinear uncertain systems," International Journal of Modelling, Identification and Control, vol. 29, no. 2, 2018.

[9] Efrain Ibarra and Pedro Castillo, "Nonlinear super twisting algorithm for UAV attitude stabilization," in 2017 International Conference on Unmanned Aircraft Systems (ICUAS), Miami USA, June 2017, pp. 640645 .

[10] Jinkun Liu and Xinhua Wang, Advanced Sliding Mode Control for Mechanical Systems: Design, Analysis and MATLAB Simulation, SpringerVerlag, London, 2006.

[11] Antonio Visioli, Practical PID Contro, Springer Berlin Heidelberg, Berlin, 2011. 